\title{
Crack initiation patterns at electrode edges in multilayer ferroelectric actuators
}

\author{
Amir Abdollahi and Irene Arias \\ Laboratori de Càlcul Numèric (LaCàN), Departament de Matemàtica Aplicada III, \\ Universitat Politècnica de Catalunya (UPC), Campus Nord UPC-C2, E-08034 \\ Barcelona, Spain \\ E-mail: irene.arias@upc.edu
}

\begin{abstract}
In multilayer ferroelectric actuators, electrode edges are the main source of fracture due to the generation of non-uniform electric fields in their vicinity. The electric fields, in turn, induce incompatible strain fields and hence concentrated stresses, which may cause the ceramic to crack. In this paper, the crack initiation from the electrode edges is simulated using a phase-field model. This model is based on variational formulations of brittle crack propagation and domain evolution in ferroelectric materials. The simulation results show different crack initiation patterns depending on the bonding conditions between the ceramic and electrode layers. Three extreme conditions are considered, which are the fully cofired, partially cofired, and separated layers. The crack initiation patterns can be either delimitation along the electrode-ceramic interface or oblique cracking from the electrode into the material. The calculations suggest a mechanism explaining the experimentally observed crack branches near the electrode edges. The effects of the ceramic layer thickness and length of the internal electrode on the crack initiation are also evaluated.
\end{abstract}




\section{Introduction}

Multilayer ferroelectric actuators are ideal candidates for numerous applications in smart structures and adaptive systems in view of their small sizes, low driving voltages and quick response times. Applications include microprecision cutting machines, inkjet printer heads, laser printers, optical disk drives and laser tuning (Uchino \& Takahashi 1996, Pritchard et al. 2001), to mention a few. However, the inherent brittleness of ferroelectrics is a serious obstacle to their reliable operation in devices. In addition, the structures of multilayer actuators often employ internal electrodes, which terminate inside the ferroelectric ceramic. In the vicinity of each terminated electrode edge, a non-uniform electric field is induced by the driving voltage, producing an incompatible strain field. A complex stress field arises from this incompatibility, which may lead to crack initiation and propagation around the electrode edge. Therefore, it is necessary to understand the fracture behavior of multilayer ferroelectric actuators to assure optimum reliability of the systems and guide the design. Towards this goal, numerous experimental and theoretical investigations have been carried out

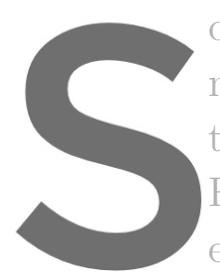
during the past decades. Experimental observations confirm that the cracks initiate
mainly from the eledtrode edge and they can propagate through the ceramic, along
the ceramic-electrode interface and perpendicular to the electrode edge (Uchino \&
Furuta 1992, Furuta \& Uchino 1993, Aburatani et al. 1994, Lucato et al. 2001, Kol
et al. 2004, Liu et al. 2005, Zhao et al. 201d). Electric-field induced crack growth of

ferroelectric ceramics has been also observed in other experiments (Lynch et al. 1995,

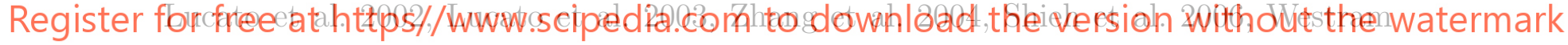
et al. 2007, Westrain et al. 2007, Beom et al. 2009, Jiang et al. 2009). Early theoretical models were developed for fracture mechanics analysis of multilayer electrostrictive ceramics (Winzer et al. 1989, Yang \& Suo 1994, Hao et al. 1996, Gong \& Suo 1996, Ru et al. 1998). Related studies have been also conducted based on the linear theory of piezoelectricity $(\mathrm{Ru}$ 2000, Ye \& He 2001, Li \& Tang 2003, Wang \& Mai 2005, Guo et al. 2006). However, the intrinsic nature of most piezoelectric materials demands the consideration of nonlinear electromechanical effects. To address this problem, nonlinear approaches were proposed taking into account the ferroelectric and ferroelastic behaviors (Kamlah \& Bohle 2001, Lucato et al. 2001, Elhadrouz et al. 2006, Zhao et al. 2010). Comprehensive reviews of related modeling approaches have been presented in (Kamlah 2001), (Landis 2004b) and (Huber 2005).

The above mentioned models for the fracture of multilayer actuators are useful to analyze the electromechanical fields near the electrode edge. Based on this analysis, some design criteria can be proposed regarding the geometry of the actuators and electrodes to reduce the probability of fracture from the electrode edge. However, 
these models do not study the crack propagation mechanisms of the actuators and these mechanisms are still unclear due to the complex interactions between the propagating cracks, electromechanical fields and microstructure of the material near the electrode edge. We have recently introduced phase-field models for the coupled microstructure and fracture evolution in ferroelectric single and polycrystals (Abdollahi \& Arias 2011a, Abdollahi \& Arias 2011b, Abdollahi \& Arias 2012a, Abdollahi \& Arias 2012b). The potential of this phase-field approach to capture the complex interactions between the crack and the material microstructure motivates us to employ it for the fracture analysis of multilayer ferroelectric actuators. In particular, the objective of this paper is to study the crack initiation at the electrode edge during the poling process. Considering different bonding conditions between the ceramic and electrode layers, different crack initiation patterns are obtained, which are useful to understand the fracture processes in this type of actuators.

The structure of the paper is as follows. The theory of the phase-field model is summarized in section 2. Numerical simulations are presented in section 3, along with a discussion of the observed crack initiation patterns and fracture mechanisms. The last

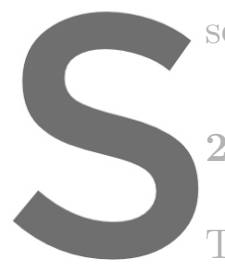
section is the conclusion

.
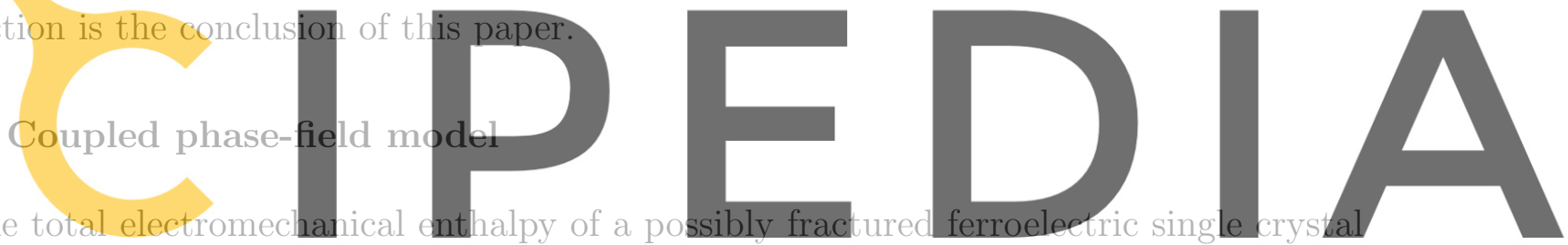

occupying a region $\Omega$ is stated in terms of the mechanical displacement $\boldsymbol{u}$, the

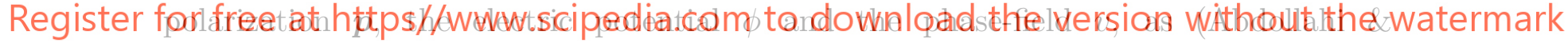

Arias 2011a, Abdollahi \& Arias 2011b)

$$
\begin{aligned}
H[\boldsymbol{u}, \boldsymbol{p}, \phi, v]= & \int_{\Omega}\left[W_{e}(\varepsilon(\boldsymbol{u}), v)+W_{f}(\varepsilon(\boldsymbol{u}), \boldsymbol{p}, \phi, v)\right] \mathrm{d} \Omega \\
& +G_{c} \int_{\Omega}\left[\frac{(1-v)^{2}}{4 \kappa}+\kappa|\nabla v|^{2}\right] \mathrm{d} \Omega,
\end{aligned}
$$

where body loads, volume charges, tractions and surface charges have been ignored for simplicity. The first integral is referred to as total bulk energy of the material, where $W_{e}$ is the part of the bulk energy density associated with the strain $\varepsilon$ and $W_{f}$ is the electromechanical energy density associated with the ferroelectric response. The second integral takes the role of the surface energy, where $G_{c}$ is the critical energy release rate or the surface energy density in Griffith's theory (Griffith 1921). The scalar field $v$ is the phase-field parameter describing a smooth transition in space between unbroken $(v=1)$ and broken $(v=0)$ states of the material. $\kappa$ is a positive regularization constant which regulates the size of the smeared fracture zone. The energy density $W_{e}$ is written as 


$$
W_{e}(\varepsilon, v)=\kappa_{0} \frac{\operatorname{tr}^{-}(\varepsilon)^{2}}{2}+\left(v^{2}+\eta_{\kappa}\right)\left(\kappa_{0} \frac{\operatorname{tr}^{+}(\varepsilon)^{2}}{2}+\mu \varepsilon_{D} \cdot \varepsilon_{D}\right),
$$

where $\kappa_{0}$ and $\mu$ are the bulk and shear modulus of the material, respectively. The trace

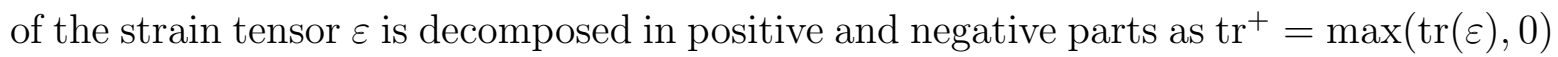
and $\operatorname{tr}^{-}=\max (-\operatorname{tr}(\varepsilon), 0)$ and $\varepsilon_{D}$ are the deviatoric components of the strain tensor. This decomposition is introduced to distinguish the contributions to the strain energy due to compression, expansion, and shear. To prevent crack nucleation, propagation and interpenetration in compressed regions, only the expansion and shear terms are multiplied by the jump set function $\left(v^{2}+\eta_{\kappa}\right)$ (Amor et al. 2009). This condition is essential for the simulation of the crack propagation at the electrode edge since high compressive stresses are induced in this region (Furuta \& Uchino 1993, Kamlah \& Bohle 2001). The parameter $\eta_{\kappa}$ is a small (relative to $\kappa$ ) residual stiffness to avoid the singularity of the bulk energy in fully fractured regions of the domain.

The electromechanical energy density $W_{f}$ associated with polarization $p$, electric potential $\phi, \varepsilon$ and $v$ is given as

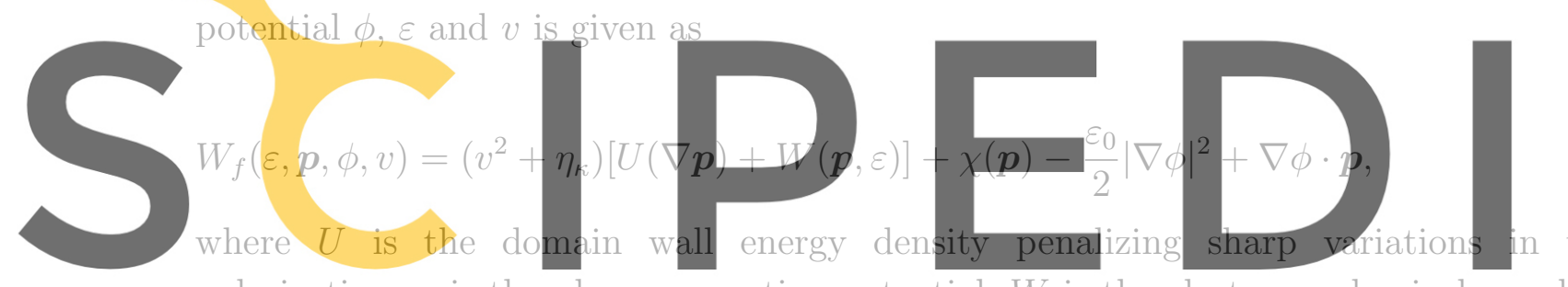

polarization, $\chi$ is the phase separation potential, $W$ is the electro-mechanical coupling

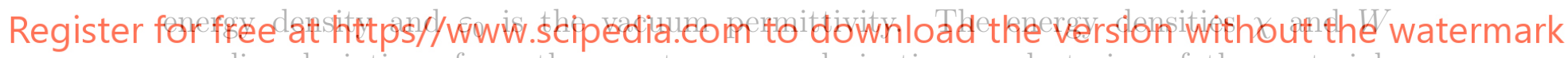
penalize deviations from the spontaneous polarizations and strains of the material, hence introducing the anisotropy and nonlinearity of ferroelectric materials. The combination of the energy functions $\chi, W$ and $W_{e}$ is the total Landau-Devonshire energy density furnishing a multi-well energy landscape with four minima (in two dimensions) corresponding to the four variants of the tetragonal phase. The detailed formulation of these energy functions and the related material constants are presented in Appendix A.

In addition to the asymmetric fracture response in tension and compression, this particular formulation of the phase-field model encodes the following assumed crack conditions: (1) traction-free, (2) electrically permeable, and (3) free-polarization (Abdollahi \& Arias 2011b). The permeable conditions assume that crack faces are closed and the electrical fields are not perturbed by the presence of the crack. These conditions have been checked to be realistic based on the fracture behavior of metal-piezoceramic interfaces in commercial multilayer actuators (Haeusler et al. 2009). Theoretical studies also show that the permeable conditions are a reasonable approximation when the electrical discharge strength of the medium within the crack is small, in the order of that of air (Landis 2004a, Li et al. 2008). In addition, free-polarization boundary conditions 
are commonly assumed, implying that the polarization distribution is unaffected by the presence of the crack, and hence dictated by the bulk material model (Wang \& Kamlah 2010). We refer to (Abdollahi \& Arias 2011a, Abdollahi \& Arias 2012b) for further details and implementation of these conditions for diffuse cracks in the context of the phase-field models.

The time evolution of the system results from the gradient flows of the total electromechanical enthalpy with respect the primary order parameters. Here, the primary order variables are $\boldsymbol{p}$ and $v$ for the microstructure and fracture processes, respectively. Thus, the governing equations are obtained by assuming that the displacement and the electric field adjust immediately to mechanical and electrostatic equilibrium (with infinite mobility), i.e.

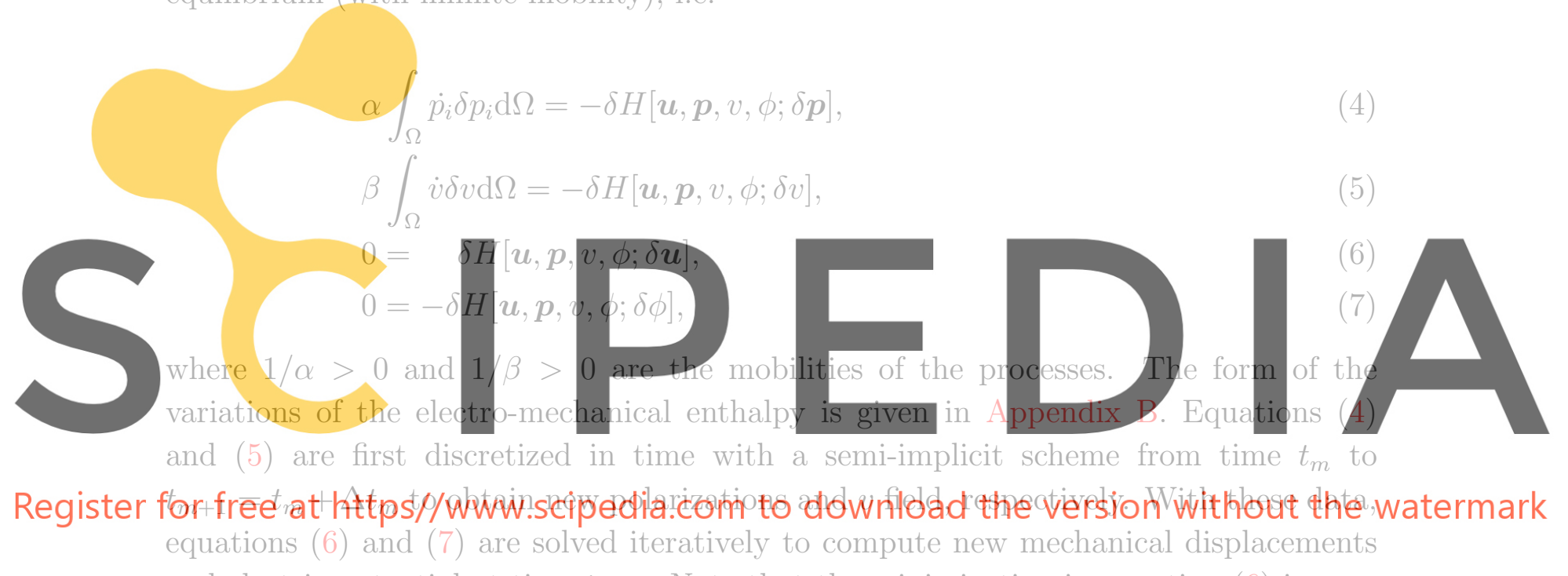

and electric potential at time $t_{m+1}$. Note that the minimization in equation (6) is non-

smooth, and a quasi-Newton algorithm is applied to solve the mechanical equilibrium problem following (Amor et al. 2009).

Two constraints are also considered for solving equations (4) and (5). The first constraint imposes an irreversibility condition for the fracture process in equation (5), namely that the field $v$ can only decrease at any point in space during the incremental process. When the value of $v$ decreases beyond a threshold $\gamma$, it is forced to zero for the rest of the calculation. The second constraint puts an upper bound on the magnitude of the polarization in equation (4). Since the driving voltage in multilayer ferroelectric actuators generates a high electric field at the electrode edge, the polarization magnitude may reach a critical value $p_{c}$ in this region. This level of polarization is induced by a certain level of electric field, called dielectric strength, above which the ferroelectric ceramic experiences failure of its insulating properties, i.e. electrical breakdown. This value is commonly measured as the average breakdown strength of thick-film ferroelectric samples, which underestimates the magnitude of the high electric field at the electrode 
edge (Burn \& Smyth 1972, Young et al. 2007). In the absence of precise information about this magnitude, we choose the maximum value for $p_{c}$ such that no instabilities on the polarization are observed near the electrode edge as a result of the high electric field. Similar constraints have been introduced in the literature based on the concepts of dielectric breakdown, which are a strip dielectric breakdown (DB) (Zhang \& Gao 2004, Gao et al. 2006), and a polarization saturation (PS) (Gao \& Barnett 1996) models.

\section{Numerical simulations}

\subsection{Computational domain}

A schematic of a multilayer actuator is presented in figure 1(a). Due to the symmetry, only half of a single layer highlighted by the hatched area, is considered for the simulation. This area is indicated in figure $1(\mathrm{~b})$ as the computational model. The boundary conditions of this model are as follows. Along the top electrode, the electric

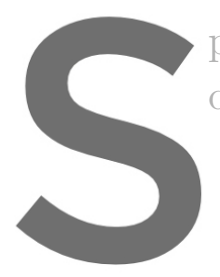
potential is fixed to tero and the deformation of the la

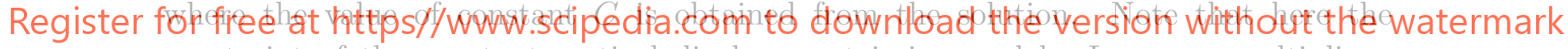 constraint of the constant vertical displacement is imposed by Lagrange multipliers.} The right electrode is connected to the ground and is traction-free:

$$
\phi\left(L_{2}, y\right)=0, \quad \sigma_{x x}=\sigma_{x y}=0 .
$$

The left boundary is along the vertical line of symmetry, therefore the shear stress and the horizontal components of mechanical and electric displacements vanish:

$$
D_{x}\left(-L_{1}, y\right)=0, \quad u_{x}\left(-L_{1}, y\right)=0, \quad \sigma_{x y}=0 .
$$

The bottom boundary consists of two interfaces between (1) ceramic-electrode and (2) ceramic-ceramic layers. Here we consider three extreme assumptions for the bonding of these layers. The first condition assumes that the ceramic layers and internal electrodes are firmly cofired together without considering any defects between the layers, i.e. the fully cofired model. As an intermediate assumption, the ceramic-electrode interface is considered to be fully cofired while there is no ceramic-ceramic bonding ahead of the 


\section{external electrode}

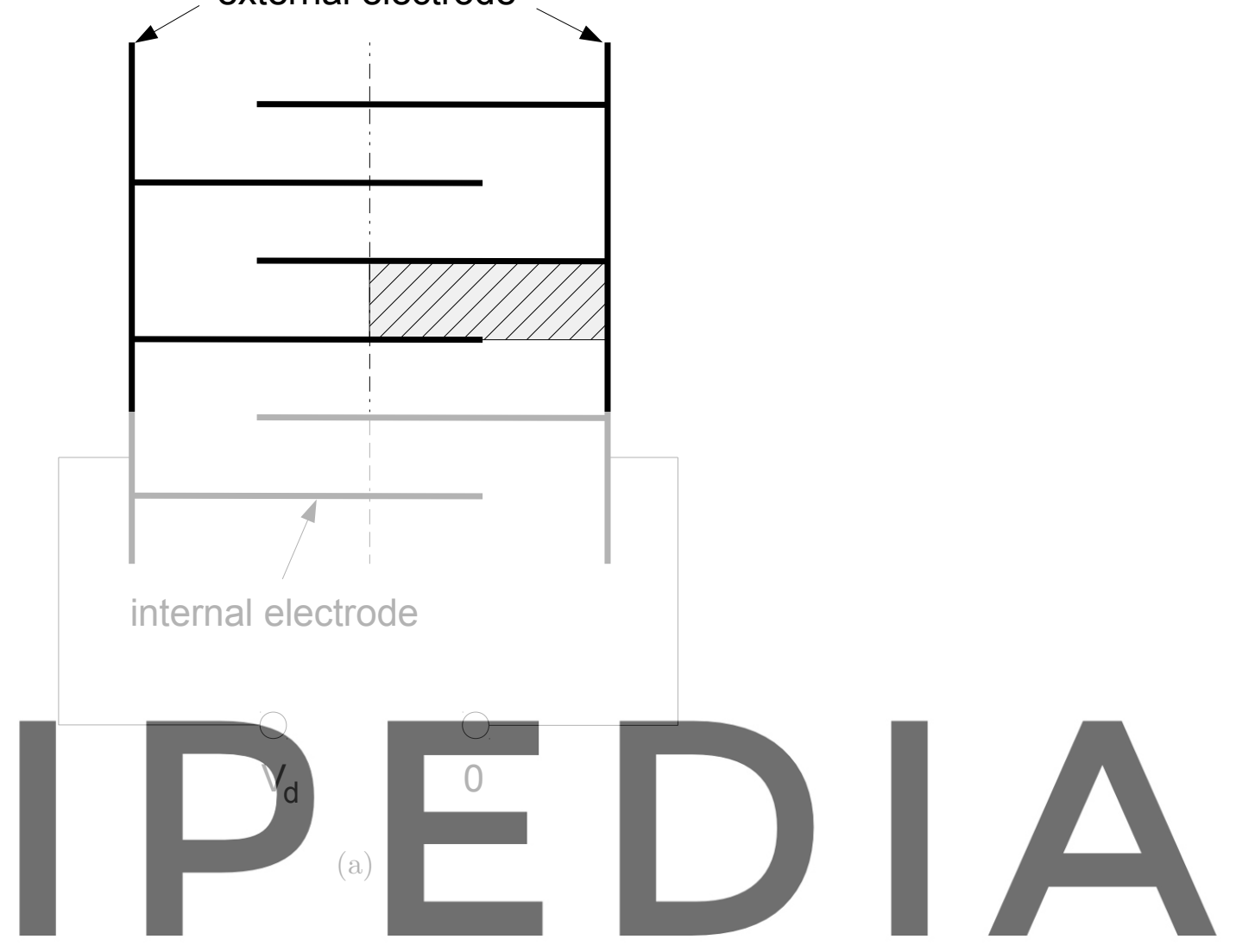

Register for free at https//www.scipedia.com to download the version without the watermark

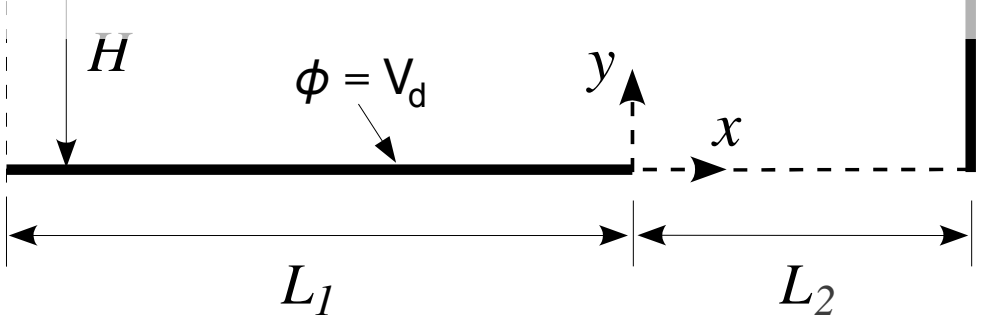

(b)

Figure 1. (a) Schematic of a multilayer actuator (b) Schematic of the computational model. The electrodes are indicated with the thick lines and the origin of the coordinate system is located at the electrode edge. 
electrode edge (Zhao et al. 2010). This model is called the partially cofired model. Finally, the third assumption considers fully separated layers on both interfaces. In fact this model represents a single layer of the multilayer actuator where the internal electrode is located at the surface of the actuator (Lucato et al. 2001). This model is called the surface electrode model. Electrical conditions at the bottom boundary are identical for the three models:

$$
\begin{aligned}
& \phi(x, 0)=V_{d}, \quad-L_{1} \leqslant x \leqslant 0, \\
& D_{y}(x, 0)=0, \quad 0<x \leqslant L_{2},
\end{aligned}
$$

where $V_{d}$ is the driving voltage of the actuator. However these models impose different mechanical conditions at the bottom boundary as follows:

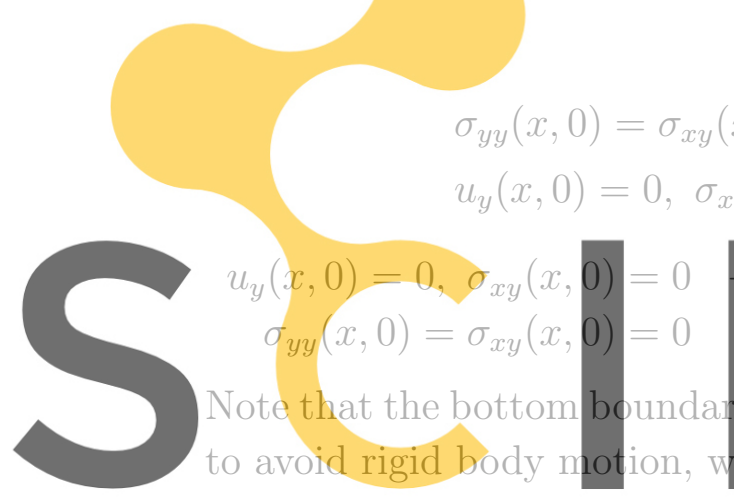

of this model.

Surface electrode model

Fully cofired model

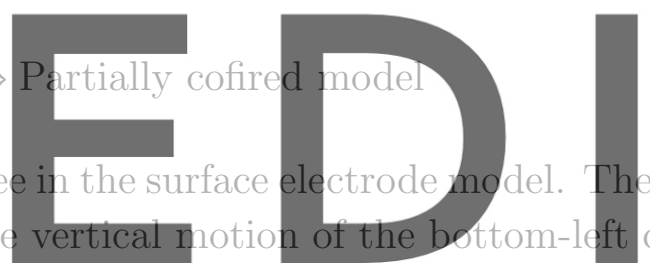

$(13)$

$(14)$

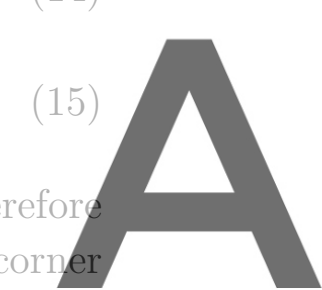

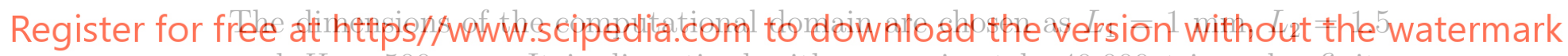

$\mathrm{mm}$ and $H=500 \mathrm{~m}$. It is discretized with approximately 40,000 triangular finite

elements of different sizes. The fine mesh with element size $h=1 \mathrm{~nm}$ is generated in a

small rectangular area of size $100 \times 100 \mathrm{~nm}^{2}$ around the electrode edge, where fracture is expected. This element size is small enough for the regularization of the ferroelectric domain walls with a thickness of few nanometers (Stemmer et al. 1995, Floquet et al. 1997). The rest of the domain is meshed with larger elements. A sample mesh is presented in figure 2. Note that in the phase-field model, a domain wall scaling parameter $a_{0}$ regulates the size of the domain walls. This parameter has to be chosen such that the variation of the polarization can be resolved by the discretization, particularly across the coarse elements away from the electrode edge, while the domain walls near the electrode edge remain sufficiently sharp in the order of their physical thickness. These conditions are met in the simulations by increasing $a_{0}$ with the element size. See Appendix A for further details on setting this parameter.

Similar to the experimental procedures, the model is polarized under a sufficiently large electric field. For the poling process, an electric field $E=V_{d} / H$ of up to 1.6 $\mathrm{KV} / \mathrm{mm}$ is applied incrementally between the electrodes. This is done by increasing the 


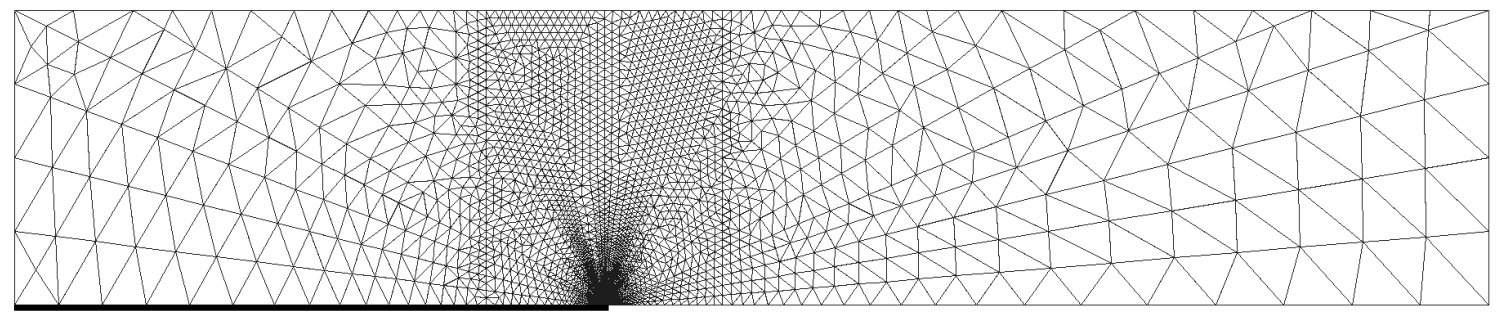

Figure 2. Sample of the meshed model where a finer mesh is generated near the electrode edge.

driving voltage $V_{d}$ through 80,000 time steps with a load function $V_{d}(t)=A t$, where $A=1 \mathrm{~V} / \mathrm{s}$, and a time step $\Delta t=10^{-2} \mathrm{~s}$. This time step also leads to convergent and accurate solutions for the time integration of gradient flow equations in equations (4) and (5). The initial data of the model are set as $\boldsymbol{p}_{0}=0, \boldsymbol{u}_{0}=\mathbf{0}, \phi_{0}=0$, and $v_{0}=1$. The remaining constants are given in Appendix A. The simulations are carried out on parallel processors using the finite element library of the Kratos multi-physics package

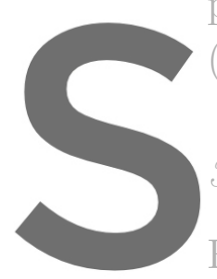
(Dadvand et al. 2010 (3.). 3.2. Numerical results and dise For the first simulations. we study the electromechanic
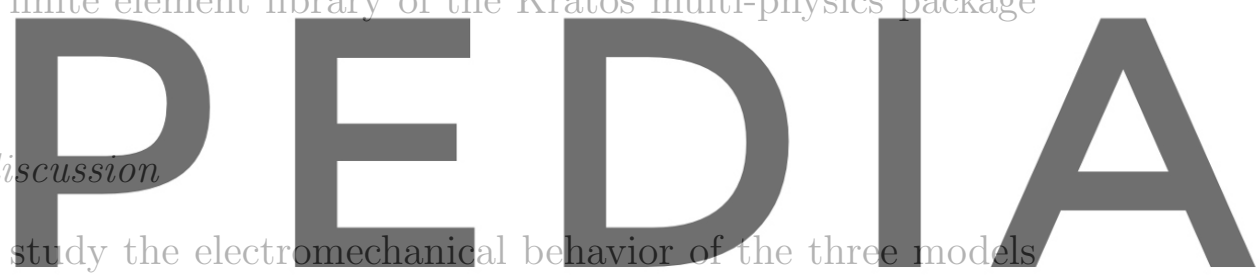

without considering the fracture process. These simulations are performed without

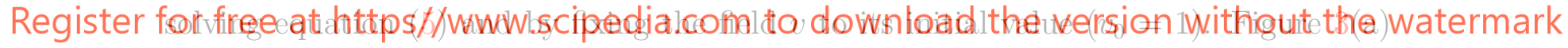

presents the distribution of the vertical polarization field $p_{y}$ in the surface electrode model after the poling process under the electric field $E=1.6 \mathrm{KV} / \mathrm{mm}$. It is clear that the active area, covered by the electrodes, is polarized along the electric field in the positive vertical direction. On the other hand, the inactive area remains almost unpolarized due to the absence of the internal electrode and a weak induced electric field in this area. Special attention should also be paid to the polarization distribution at the electrode edge due to the generation of a non-uniform and high electric field in this area. Figure 3(b) focuses on a small neighborhood of the electrode edge (fine mesh zone), where the polarization distribution shows the formation of two $90^{\circ}$ domains. The polarization in the first domain is aligned along the applied electric field between the electrodes, while in the second domain it is forced to align along the interface ahead of the electrode edge, satisfying the charge-free condition of this boundary, see equation (12). It is also obvious that the magnitude of the polarization increases near the electrode edge due to the high induced electric field in this area. We observe similar polarization distributions and domain structures in the fully and partially cofired models, which are 
omitted here for the sake of brevity.

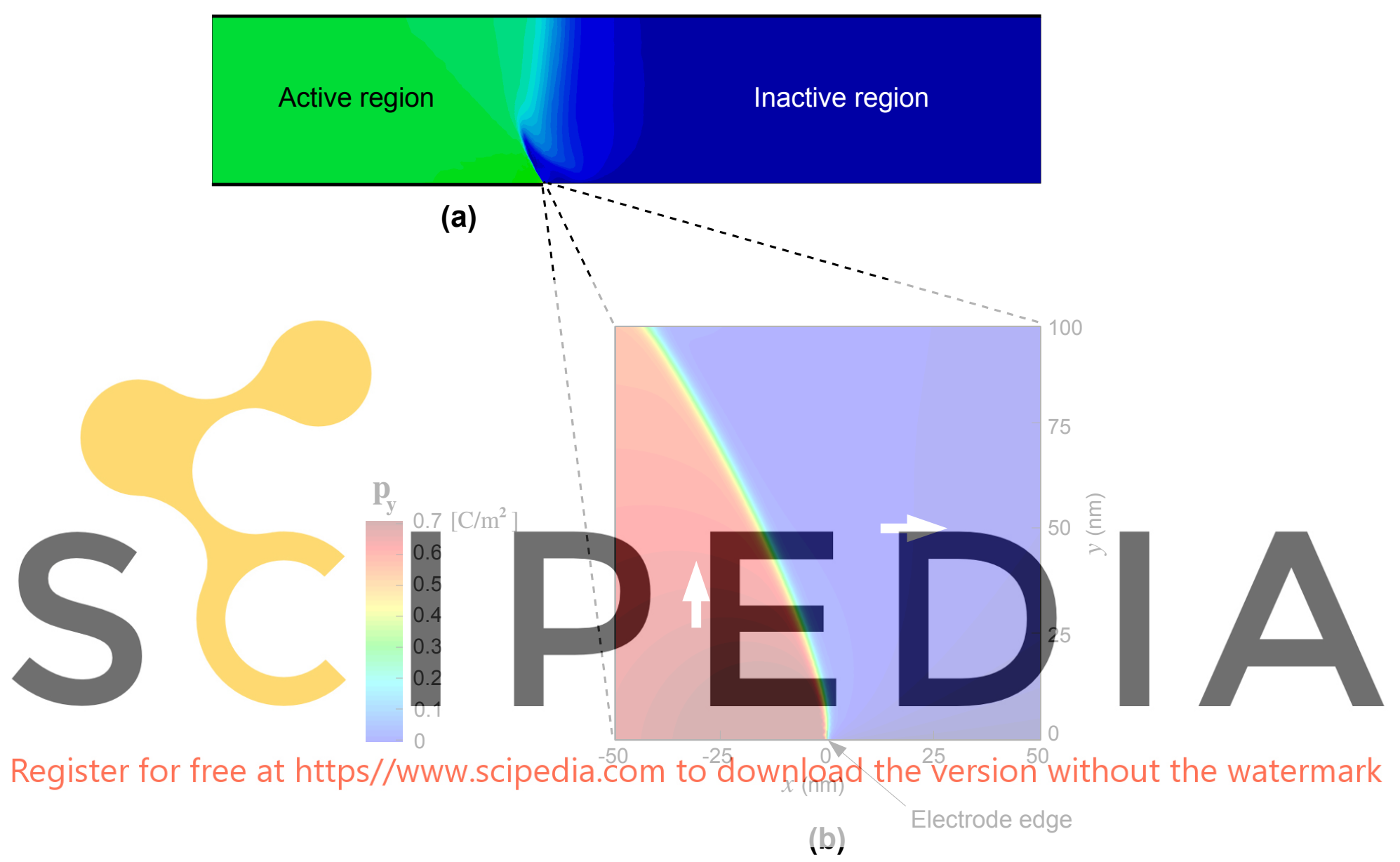

Figure 3. Distribution of the vertical polarization field $\boldsymbol{p}_{y}$ after the poling process under the electric field $E=1.6 \mathrm{KV} / \mathrm{mm}$ in (a) the surface electrode model, and (b) a small neighborhood of the electrode edge. Domain orientations are indicated with large arrows. Similar domain structures are observed in the fully and partially cofired models.

The main differences between the results of the three models are the deformation and magnitude of the polarization in the active area. Figure 4 presents the evolution of the polarization during the poling process in the top-left corner of the models. The polarization increases in a nonlinear fashion with the applied electric field until it reaches a saturation level, which is around the spontaneous polarization $0.26 \mathrm{C} / \mathrm{m}^{2}$ in the surface electrode model. The polarization evolution results in an expansion of the material in the direction of the applied electric field and a shrinkage perpendicular to the field in the active area. This is obvious from the deformed configuration of the surface electrode 
model in figure 5(c). The inactive area mechanically clamps the active part of the material, which leads to a strain mismatch and high stresses near the electrode edge. In the partially cofired model, the mechanical constraint of the internal electrode increases the stresses in the active area which act against the applied electric field and result in a partial mechanical depolarization of this area. Consequently, the saturation level of the polarization in figure 4 and the vertical mechanical displacement of the upper electrode in figure 5(b) decrease slightly with respect to the surface electrode model. These differences are more significant in the fully cofired model where the whole bottom boundary is mechanically constrained in the vertical direction, see figure 4 and figure $5(\mathrm{a})$.

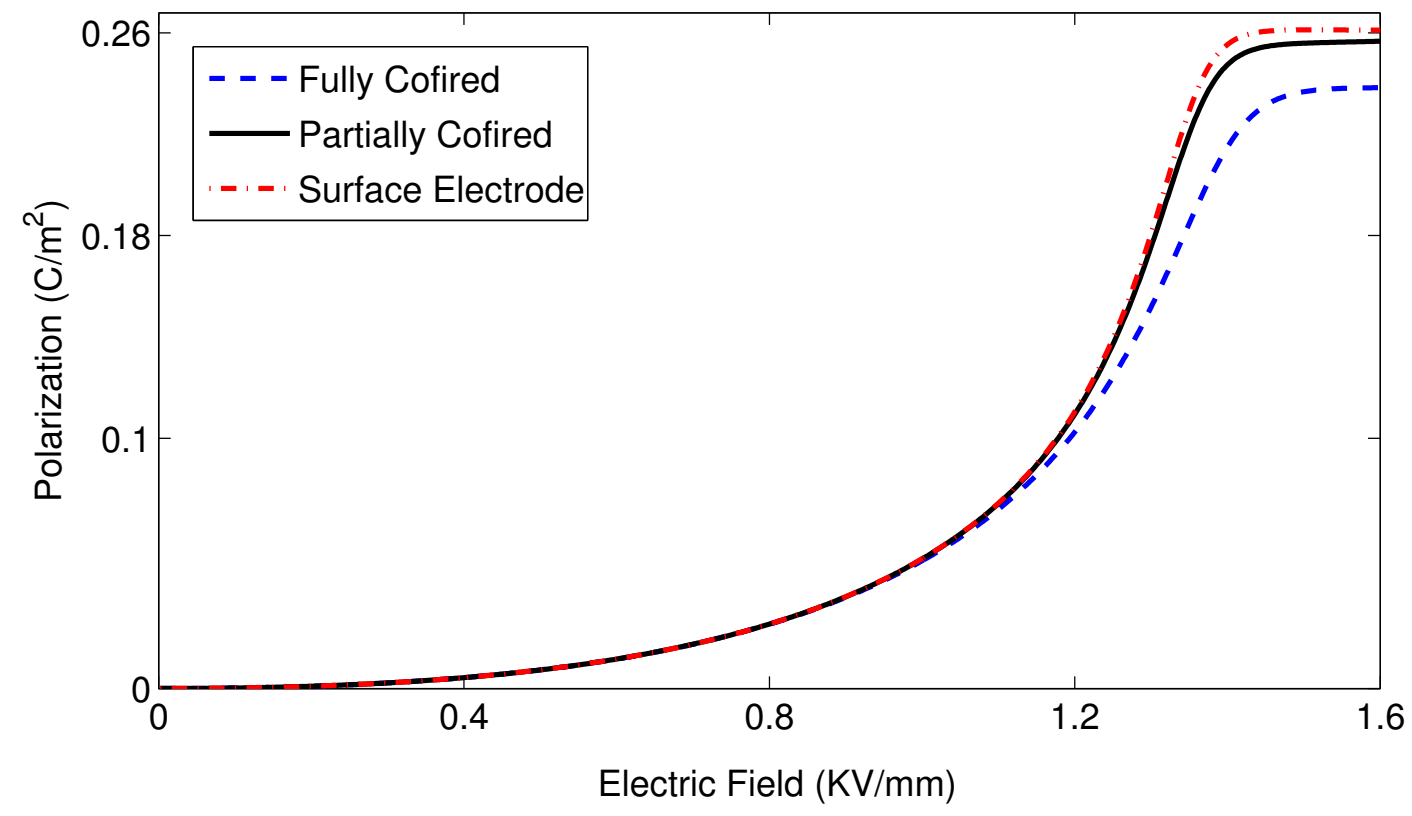

Figure 4. Evolution of the polarization as a function of the applied electric field during the poling process in the top-left corner of the fully cofired, partially cofired and surface electrode models.

The deformed configurations presented in figure 5 result in different stress field distributions at the electrode edge, and hence different crack initiation patterns. To obtain these patterns, the simulations are performed by also solving equation (5) for the fracture process. Figure 6 presents snapshots of the crack propagation in a small neighborhood of the electrode edge for the three models. In all of the simulations, as the electric field increases, the $v$ field starts to decrease at the electrode edge until it reaches 


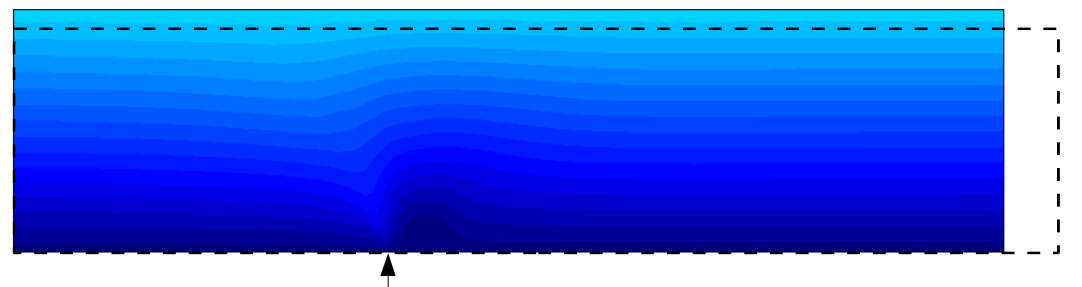

Electrode edge

(a)

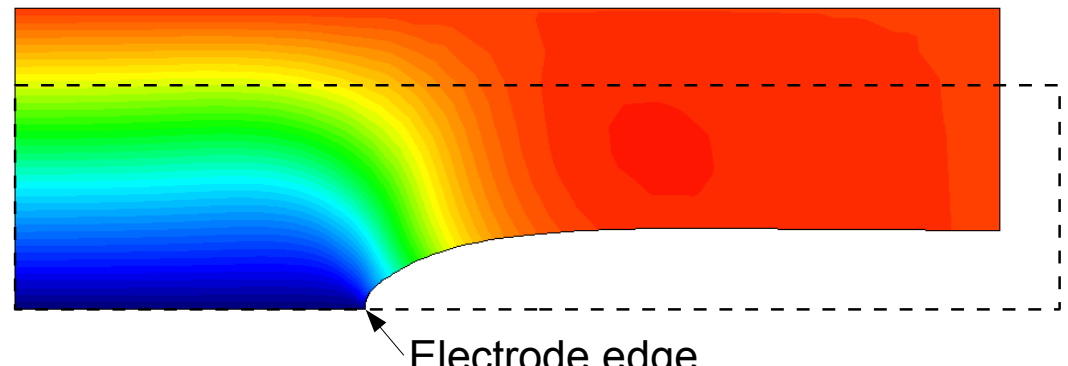

(b)

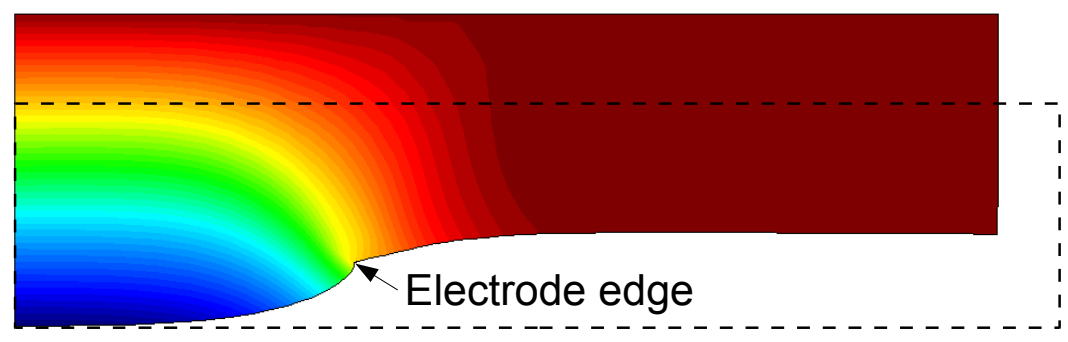

$u_{y}$

(c)

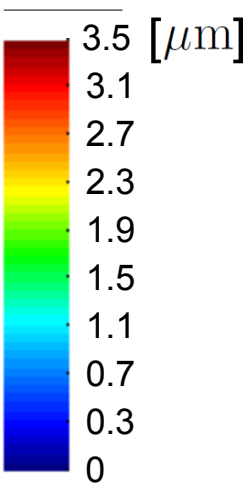

Figure 5. Deformed configuration of the (a) fully cofired, (b) partially cofired, and (c) surface electrode models after the poling process. The color contour indicates the distribution of vertical displacement $\boldsymbol{u}_{y}$. The deformation is exaggerated for clarity with a scale factor of 50 . The position of the electrode edge is indicated with the arrow and the border of the undeformed configuration is highlighted with the dashed line. 
the threshold to be considered permanently fractured $(v<0.02)$. By further increasing of the electric field, the $v$ field evolves in a different direction from the electrode edge in each model. Figure 6(a) shows that the crack mainly propagates along the ceramicceramic interface ahead of the electrode edge in the fully cofired model. On the contrary, figure 6(b) indicates that a preferential path for the crack propagation is along the ceramic-electrode interface in the partially cofired model. It is noteworthy that the experimental results in (Zhao et al. 2010) show a similar crack propagation path along the electrode-ceramic interface in a partially cofired actuator. This fracture process can be easily understood as follows. The interface ahead of the electrode edge is traction-free and it opens under an applied electric field (see figure 5(b)). Therefore this interface gap behaves similar to a pre-crack in the model which facilitates the crack propagation along the electrode-ceramic interface. As for the surface electrode model, it is interesting to observe in figure 6(c) that the crack mainly propagates along the $90^{\circ}$ domain wall, separating the $90^{\circ}$ domains in figure $3(\mathrm{~b})$. Since the electrode is also traction-free in this model, the surface deformation ahead of the electrode edge cannot induce a high stress concentration along the electrode-ceramic interface. In this condition, stresses are dominant within the domain wall, provided a favorable path for the crack propagation.

The snapshots of the crack propagation in figures 6(a)-(c) correspond to applied electric fields $E=1.1,0.6$ and $1.6 \mathrm{KV} / \mathrm{mm}$, respectively. The simulations are stopped at these load steps because by further increasing of the load, the cracks propagate though the coarse mesh zone, where they cannot be resolved numerically. More extended cracks can be obtained by employing an adaptive mesh refinement strategy, which in turn magnifies the computational cost of the simulations. However, the models proposed here target a mesoscale crack propagation and domain formation at the electrode edge and the extension to the larger scale is beyond the scope of the present paper. The corresponding loads of the crack initiation in figure 6 suggest that fracture is more likely to initiate in a partially cofired actuator than a fully cofired one under the same electrical load. The highest reliability is found in the surface electrode model, where the crack initiates under a high electric field after the poling process. We also note that in the three models, the propagating crack does not affect the domain formation near the electrode edge considerably. This is due to the fact that the domain formation is mainly governed by the high electric field in this region rather than the stresses around the crack tip. Furthermore, the permeable crack does not constitute an electrical barrier for the distribution of the electrical fields.

The crack initiation patterns presented in figure 6 also suggest a crack propagation scenario in multilayer ferroelectric actuators. The most common design of these 


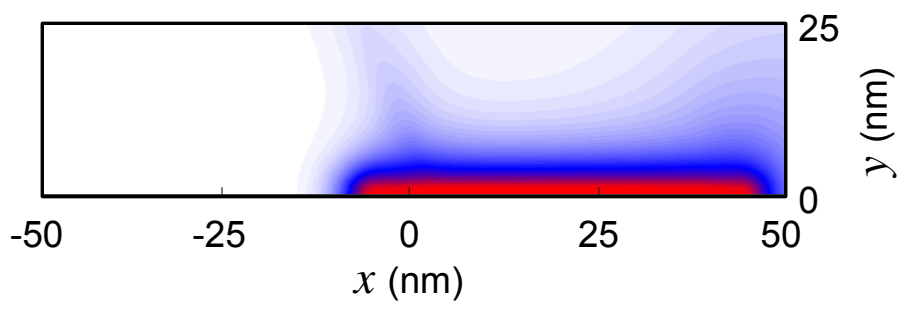

(a)

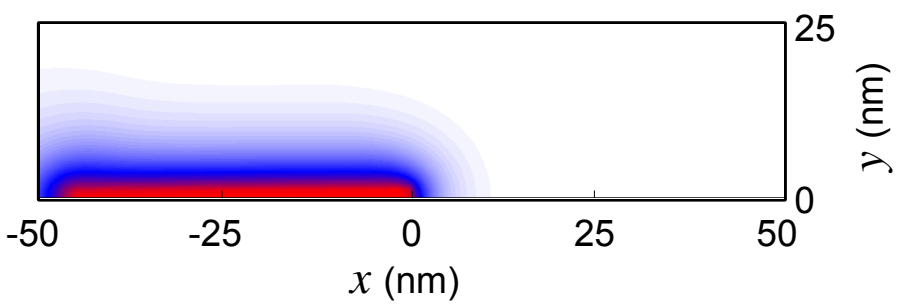

(b)

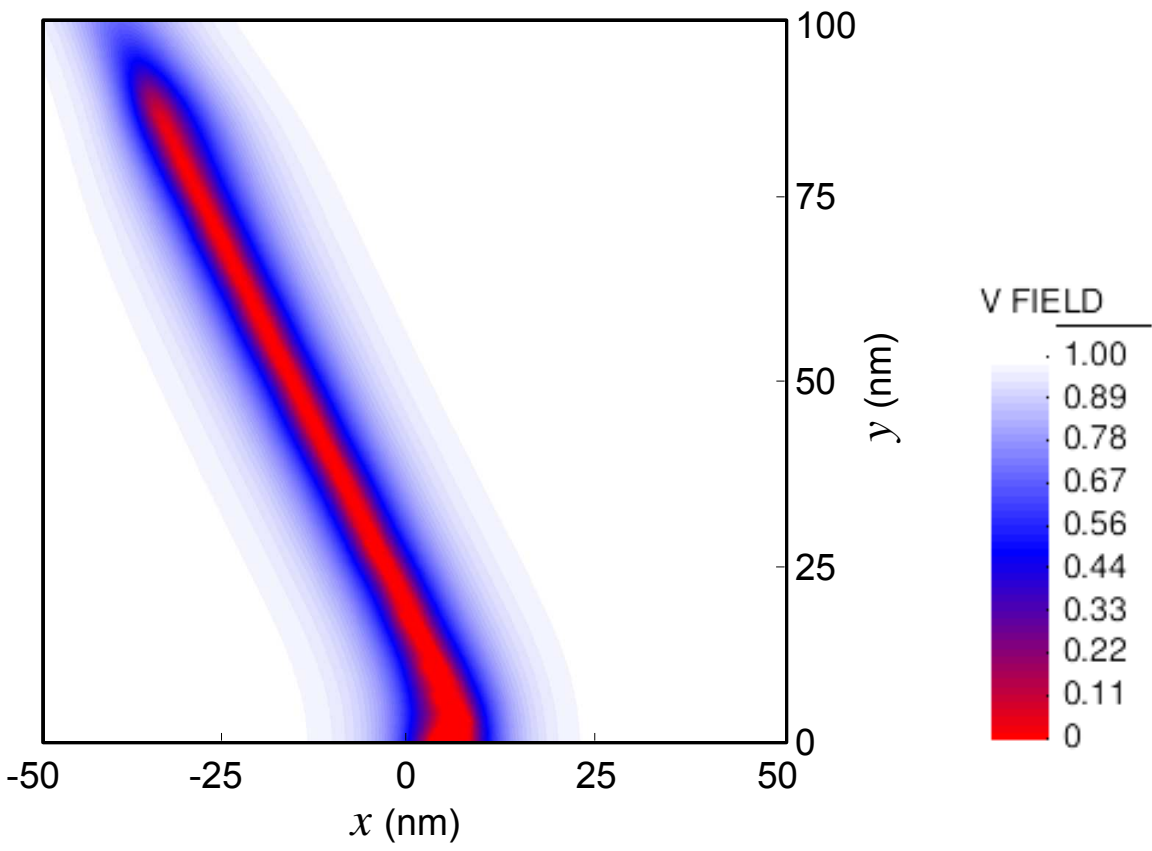

(c)

Figure 6. Crack initiation patterns in a small neighborhood of the electrode edge for the (a) fully cofired, (b) partially cofired, and (c) surface electrode models under electric fields $E=1.1,0.6$ and $1.6 \mathrm{KV} / \mathrm{mm}$, respectively. The color contour indicates the distribution of $v$ field representing the fracture area. Recall that the origin of the coordinate system is located at the electrode edge. 
actuators is that the ceramic layers and electrodes are fully cofired together. For this design, the simulation results show that the first crack initiation site is the ceramic-ceramic interface ahead of the electrode edge, see figure 6(a). If the crack propagates a certain distance along this interface, a new crack will initiate in the opposite direction along the electrode-ceramic interface, as also observed in figure 6(a). This fracture process can be explained as follows. Since the first crack gradually cuts the ceramic-ceramic interface into a traction-free surface, an interface gap is formed ahead of the electrode edge and the fully cofired actuator becomes a partially cofired one. Consequently, in the partially cofired actuator, the new crack initiates along the electrode-ceramic interface, as presented in figure 6(b). A sufficient propagation of the new crack changes the electrode-ceramic interface into a traction-free surface and the partially cofired actuator gradually converts to a surface electrode one. In this situation, the third mode of fracture will be activated, which is the crack propagation along the $90^{\circ}$ domain wall in the surface electrode model, see figure $6(\mathrm{c})$. The above mentioned events reflect a crack initiation process in multilayer ferroelectric actuators, which is schematically presented in figure 7. Interestingly, the experimental results show that the crack is initiated at the internal electrode edge, and it branches basically into three directions from the electrode edge which results in the delamination and oblique cracking of multilayer ferroelectric actuators (Uchino \& Furuta 1992, Furuta \& Uchino 1993, Aburatani et al. 1994). Our calculations suggests a mechanism explaining these observations. Note that far from these initiation sources, the direction of crack propagation can be affected by the presence of pores, domain walls or grain boundaries in polycrystals.

We also evaluate the effects of the ceramic layer thickness and length of the internal electrode on the crack initiation. By changing these dimensions, we observe significant effects in the surface electrode model. Therefore this model is considered in the following simulations. First, the simulations are performed for a thinner ceramic layer by decreasing the thickness of the model from $H=500 \mu \mathrm{m}$ to $H=125 \mu \mathrm{m}$. The value of the driving voltage $V_{d}$ is also decreased proportionally to apply the same electric field of the first simulations, i.e. $A=0.25 \mathrm{~V} / \mathrm{s}$. Simulation results show that after the poling process, the $v$ field reaches a minimum value of 0.85 at the electrode edge, i.e. the crack does not initiate, while under the same load, the crack initiates along the domain wall in the thick layer model, see figure 6(c). Therefore the probability of fracture decreases significantly by decreasing the thickness of the ceramic layer. This is in agreement with experimental observations of surface electrode actuators, where numerous cracks are found perpendicular to the electrode edge in thick specimens and only a few in thin ones (Lucato et al. 2001). 


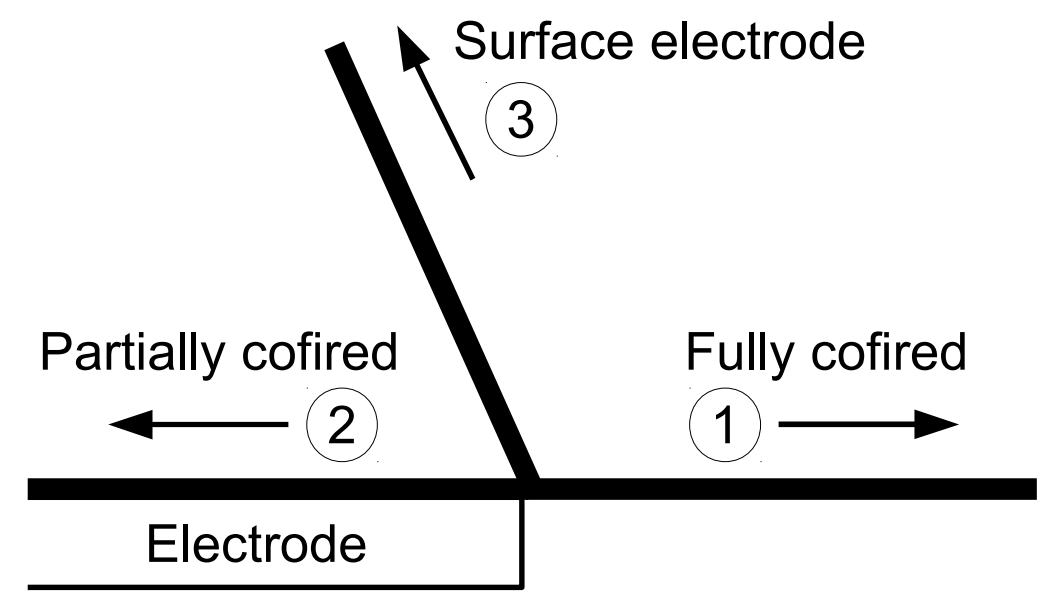

Figure 7. Schematic of the crack initiation events in multilayer ferroelectric actuators. The thick line is a schematic of the crack path and the arrows present the crack propagation directions from the electrode edge. The numbers indicate the sequence of crack initiation events from the fully cofired to the surface electrode model.

Next, we perform simulations with a longer internal electrode by choosing $L_{1}=$ $2 \mathrm{~mm}$ and $L_{2}=0.5 \mathrm{~mm}$. A similar crack initiation pattern of figure 6(c) is observed under a lower electric field $E=1.4 \mathrm{KV} / \mathrm{mm}$. Therefore, increasing the coverage of the internal electrode results in an increase in the crack length under the same electric load. Experiments also confirm that the lengths of starter cracks at the electrode edge are longer in wide electrodes with respect to narrow ones (Lucato et al. 2001).

Note that similar effects of the layer thickness and electrode coverage are observed in the fully and partially cofired models, although they are less pronounced in these models with respect to the surface electrode one.

\section{Conclusions}

We perform, to the best of our knowledge, the first simulations of crack initiation and propagation at electrode edges in multilayer ferroelectric actuators, which suggest a mechanism for failure in these devices. The phase-field model is based on variational formulations of brittle crack propagation and domain evolution in ferroelectric materials. The simulation results show different crack initiation patterns depending on the bonding conditions of the ceramic and electrode layers. For fully cofired layers, the crack mainly propagates through the ceramic ahead of the electrode edge. If the ceramic layers are partially cofired with the electrode, the crack initiates along the ceramic-electrode interface. For fully separated layers, which represent a surface electrode model, an oblique crack propagation is observed along the $90^{\circ}$ domain wall. 
These crack initiation events provide a mechanistic view of the fracture process in multilayer actuators. Furthermore, the obtained patterns can be interpreted as initiation sources of experimentally observed crack branches near the electrode edge (Uchino \& Furuta 1992, Furuta \& Uchino 1993, Aburatani et al. 1994).

We also evaluate the effects of the ceramic layer thickness and length of the internal electrode on the crack initiation. The results show that the crack initiation becomes more difficult by decreasing the thickness of the ceramic layer and the length of the internal electrode. A similar behavior has been observed in experiments for crack initiation and propagation perpendicular to the electrode edge (Lucato et al. 2001).

We also suggest that more work is needed to evaluate other possible effects on the fracture of multilayer actuators. In particular, dielectric breakdown often occurs via the formation of conducting tubular channels (Suo 1993), which can release a large amount of thermal energy. This energy may affect the material properties or may induce a thermal stress near the electrode edge. Therefore, a thermo-electro-mechanical analysis is required to investigate this effect. Furthermore, the formation and propagation of the tubular channels around the electrode edge can have an important effect on the electromechanical fields and the microstructure of the material. These topics are currently under investigation.

\section{Appendix A. Energy functions and model parameters}

The energy functions $U, W$ and $\chi$ in equation (3) are chosen following (Devonshire 1949, Devonshire 1951), adapted to a plane polarization and plane strain state:

$$
\begin{aligned}
& U\left(p_{i, j}\right)=\frac{a_{0}}{2}\left(p_{1,1}^{2}+p_{1,2}^{2}+p_{2,1}^{2}+p_{2,2}^{2}\right) \\
& W\left(p_{i}, \varepsilon_{j k}\right)=-\frac{b_{1}}{2}\left(\varepsilon_{11} p_{1}^{2}+\varepsilon_{22} p_{2}^{2}\right)-\frac{b_{2}}{2}\left(\varepsilon_{11} p_{2}^{2}+\varepsilon_{22} p_{1}^{2}\right)-b_{3}\left(\varepsilon_{21}+\varepsilon_{12}\right) p_{1} p_{2}, \\
& \chi\left(p_{i}\right)=\alpha_{1}\left(p_{1}^{2}+p_{2}^{2}\right)+\alpha_{11}\left(p_{1}^{4}+p_{2}^{4}\right)+\alpha_{12}\left(p_{1}^{2} p_{2}^{2}\right)+\alpha_{111}\left(p_{1}^{6}+p_{2}^{6}\right)+\alpha_{112}\left(p_{1}^{2} p_{2}^{4}+p_{2}^{2} p_{1}^{4}\right) \\
& +\alpha_{1111}\left(p_{1}^{8}+p_{2}^{8}\right)+\alpha_{1112}\left(p_{1}^{6} p_{2}^{2}+p_{2}^{6} p_{1}^{2}\right)+\alpha_{1122}\left(p_{1}^{4} p_{2}^{4}\right),
\end{aligned}
$$

where $a_{0}$ is the scaling parameter of the domain wall energy, $b_{i}(i=1,2,3)$ are the constants of the coupling terms between strain and polarization and $c_{i}(i=1,2,3)$ are the elastic constants. The phase separation energy $\chi$ is improved by adding the eighthorder terms with coefficients $\alpha_{1111}, \alpha_{1112}$ and $\alpha_{1122}$ to reproduce the dielectric behavior of barium titanate $\left(\mathrm{BaTiO}_{3}\right)$ single crystals (Li et al. 2005, Wang et al. 2007). The eighth-order term with coefficient $\alpha_{1122}$ enables the model to fit the dielectric constants 
while retaining a reasonable energy barrier for $90^{\circ}$ domain switching in the tetragonal phase (Zhang \& Bhattacharya 2005, Su \& Landis 2007). $\alpha_{1}$ is linearly dependent on temperature and its negative value makes the cubic phase unstable. $\alpha_{111}$ is estimated by fitting the spontaneous polarization of the tetragonal phase. $\alpha_{112}$ and $\alpha_{1112}$ are fitted to the dielectric permittivity perpendicular to the spontaneous polarization. $\alpha_{11}, \alpha_{12}$ and $\alpha_{1111}$ are evaluated from linear and nonlinear dielectric measurements above the Curie temperature (Wang et al. 2007). The constants are chosen to fit the behavior of single crystals of barium titanate $\left(\mathrm{BaTiO}_{3}\right)$ at room temperature with a spontaneous polarization $p_{0}=0.26 \mathrm{Cm}^{-2}$, relative spontaneous strains $\varepsilon_{a}=-0.44 \%$ along a-axis and $\varepsilon_{c}=0.65 \%$ along c-axis (Zhang \& Bhattacharya 2005, Wang et al. 2007). The domain wall scaling parameter is set as $a_{0}=37 h \mathrm{Vm}^{2} \mathrm{C}^{-1}$, where $h$ is the element size. The $90^{\circ}$ domain walls width is then spanned with 4-6 elements of size $h=1 \mathrm{~nm}$ near the electrode edge in the simulations, corresponding to 4-6 nanometers, in the order of experimentally measured values in tetragonal ferroelectric ceramics (Stemmer et al. 1995, Floquet et al. 1997). Furthermore, increasing $a_{0}$ with the element size results in a smooth variation of the polarization across coarse elements away from the electrode edge.

Normalized parameters are presented in table A1 through the following normalizations: $x_{i}^{\prime}=x_{i} \sqrt{c_{0} / l_{0}} / p_{0}, \mu^{\prime}=\mu / c_{0}, \kappa_{0}^{\prime}=\kappa_{0} / c_{0}, p_{i}^{\prime}=p_{i} / p_{0}, \varepsilon_{0}^{\prime}=\varepsilon_{0} c_{0} / p_{0}^{2}$, $\phi^{\prime}=\phi / \sqrt{l_{0} c_{0}}, \alpha_{1}^{\prime}=\alpha_{1} p_{0}^{2} / c_{0}, \alpha_{11}^{\prime}=\alpha_{11} p_{0}^{4} / c_{0}, \alpha_{12}^{\prime}=\alpha_{12} p_{0}^{4} / c_{0}, \alpha_{111}^{\prime}=\alpha_{111} p_{0}^{6} / c_{0}$, $\alpha_{112}^{\prime}=\alpha_{112} p_{0}^{6} / c_{0}, \alpha_{1111}^{\prime}=\alpha_{1111} p_{0}^{8} / c_{0}, \alpha_{1112}^{\prime}=\alpha_{1112} p_{0}^{8} / c_{0}, \alpha_{1122}^{\prime}=\alpha_{1122} p_{0}^{8} / c_{0}, b_{i}^{\prime}=b_{i} p_{0}^{2} / c_{0}$ and $c_{i}^{\prime}=c_{i} / c_{0}$, where $i=1,2,3, c_{0}=1 \mathrm{GPa}$ and $l_{0}=3.7 \times 10^{-9} \mathrm{Vm}^{3} \mathrm{C}^{-1}$. Considering Young's modulus and Poisson's ratio for $\mathrm{BaTiO}_{3}$ as $E=100 \mathrm{GPa}$ and $\nu=0.37$, respectively, the value of the critical energy release rate in plane strain is obtained as $G_{c}=\left(1-\nu^{2}\right) K_{c}^{2} / E=2 \mathrm{Jm}^{-2}$. The value of normalized critical energy release rate is then calculated as $G_{c}^{\prime}=G_{c} \sqrt{1 / l_{0} c_{0}} / p_{0}=4$. The inverse of the mobilities are chosen as $\alpha=\beta=1$. The regularization parameter $\kappa$ is set to twice the finest element size as $\kappa$ $=2 \mathrm{~nm}$, i.e. $h / \kappa=0.5$, and the threshold to detect the irreversibly fractured regions is $\gamma=2 \times 10^{-2}$. A critical polarization $p_{c}=0.7 \mathrm{Cm}^{-2}$ is imposed on the polarization evolution in equation (4).

Table A1. Normalized parameters

\begin{tabular}{ccccccccc}
\hline$\kappa_{0}^{\prime}$ & $\mu^{\prime}$ & $b_{1}^{\prime}$ & $b_{2}^{\prime}$ & $b_{3}^{\prime}$ & $\alpha_{1}^{\prime}$ & $\alpha_{11}^{\prime}$ & \\
\hline 146 & 36 & 1.4282 & -0.185 & 0.8066 & -0.0023 & -0.0029 & \\
\hline$\alpha_{12}^{\prime}$ & $\alpha_{111}^{\prime}$ & $\alpha_{112}^{\prime}$ & $\alpha_{1111}^{\prime}$ & $\alpha_{1112}^{\prime}$ & $\alpha_{1122}^{\prime}$ & $\varepsilon_{0}^{\prime}$ & $G_{c}^{\prime}$ \\
\hline-0.0011 & 0.003 & -0.00068 & 0.001 & 0.0093 & 1.24 & 0.131 & 4 \\
\hline
\end{tabular}




\section{Appendix B. Weak form of the governing equations}

The weak forms of the gradient flows in (4) and (5), together with the equations for mechanical and electrostatic equilibria in (6) and (7), follow from

$$
\begin{aligned}
\alpha \int_{\Omega} \dot{p}_{i} \delta p_{i} \mathrm{~d} \Omega & =-\delta H[\boldsymbol{u}, \boldsymbol{p}, v, \phi ; \delta \boldsymbol{p}] \\
& =-\int_{\Omega}\left[\left(v^{2}+\eta\right)\left(\frac{\partial U}{\partial p_{i, j}} \delta p_{i, j}+\frac{\partial W}{\partial p_{i}} \delta p_{i}\right)+\frac{\partial \chi}{\partial p_{i}} \delta p_{i}+\phi_{, i} \delta p_{i}\right] \mathrm{d} \Omega \\
\beta \int_{\Omega} \dot{v} \delta v \mathrm{~d} \Omega & =-\delta H[\boldsymbol{u}, \boldsymbol{p}, v, \phi ; \delta v] \\
& =-\int_{\Omega} \frac{\partial\left(W_{e}+W_{f}\right)}{\partial v} \delta v \mathrm{~d} \Omega-2 G_{c} \int_{\Omega}\left(\frac{v-1}{4 \kappa} \delta v+\kappa v_{, i} \delta v_{, i}\right) \mathrm{d} \Omega,
\end{aligned}
$$

$0=\delta H[\boldsymbol{u}, \boldsymbol{p}, v, \phi ; \delta \boldsymbol{u}]=\int_{\Omega} \frac{\partial\left(W_{e}+W_{f}\right)}{\partial \varepsilon_{i j}} \delta \varepsilon_{i j} \mathrm{~d} \Omega$,

$0=-\delta H[\boldsymbol{u}, \boldsymbol{p}, v, \phi ; \delta \phi]=\int_{\Omega} \frac{\partial W_{f}}{\partial E_{i}} \delta E_{i} \mathrm{~d} \Omega$,

where the elastic strain $\varepsilon_{i j}$ and electric field $E_{i}$ are associated with the mechanical displacement $u_{i}$ and the electric potential $\phi$, respectively, as $\varepsilon_{i j}=1 / 2\left(u_{i, j}+u_{j, i}\right)$ and $E_{i}=-\phi_{, i}$.

\section{Acknowledgments}

The authors gratefully acknowledge the support of the Ministerio de Ciencia e Innovación (DPI2010-19145).

\section{References}

Abdollahi A \& Arias I 2011a Acta Mater. 59, 4733-4746.

Abdollahi A \& Arias I $2011 b$ Model. Simul. Mater. Sci. Eng. 19, 074010.

Abdollahi A \& Arias I $2012 a$ Int. J. Fract. 174, 3-15.

Abdollahi A \& Arias I $2012 b$ J. Mech. Phys. Solids . Accepted for publication.

Aburatani H, Harada S, Uchino K, Furuta A \& Fuda Y 1994 JPN J. Appl. Phys. 1 33, 3091-3094.

Amor H, Marigo J J \& Maurini C 2009 J. Mech. Phys. Solids 57, 1209-1229.

Beom H G, Jeong K M, Park J Y, Lin S \& Kim G H 2009 Eng. Fract. Mech. 76, 2399-2407.

Burn I \& Smyth D M 1972 J. Mater. Sci. 7, 339-343.

Dadvand P, Rossi R \& Onate E 2010 Arch. Comput. Methods Eng. 17, 253-297.

Devonshire A F 1949 Philos. Mag. 40, 1040-1063.

Devonshire A F 1951 Philos. Mag. 42, 1065-1079. 
Elhadrouz M, Zineb T B \& Patoor E 2006 Int. J. Eng. Sci. 44, 996-1006.

Floquet N, Valot C M, Mesnier M T, Niepce J C, Normand L, Thorel A \& Kilaas R 1997 J. Phys. III 7, 1105-1128.

Furuta A \& Uchino K 1993 J. Am. Ceram. Soc. 76, 1615-1617.

Gao C F, Noda N \& Zhang T Y 2006 Int. J. Eng. Sci. 44(3-4), 256-272.

Gao H J \& Barnett D M 1996 Int. J. Fract. 79, R25-R29.

Gong X \& Suo Z 1996 J. Mech. Phys. Solids 44, 751-769.

Griffith A A 1921 Philos. Trans. Royal Soc. London A221, 163-198.

Guo X H, Fang D N, Soh A K, Kim H C \& Lee J J 2006 Acta Mech. Sinica 22, 34-39.

Haeusler C, Jelitto H, Neumeister P, Balke H \& Schneider G A 2009 Int. J. Fract. 160, 43-54.

Hao T H, Gong X \& Suo Z 1996 J. Mech. Phys. Solids 44, 23-48.

Huber J E 2005 Curr. Opin. Solid. State Mater. Sci. 9, 100-106.

Jiang Y, Zhang Y, Liu B \& Fang D 2009 Acta Mater. 57, 1630-1638.

Kamlah M 2001 Cont. Mech. Therm. 13, 219-268.

Kamlah M \& Bohle U 2001 Int. J. Solids Struct. 38, 605-633.

Koh J H, Jeong S J, Ha M S \& Song J S 2004 Ceram. Int. 30, 1863-1867.

Landis C M $2004 a$ Int. J. Solids Struct. 41(22-23), 6291-6315.

Landis C M 2004b Curr. Opin. Solid State Mater. Sci. 8, 59-69.

Li W Y, McMeeking R M \& Landis C M 2008 Eur. J. Mech. A-Solids 27(3), 285-301.

Li X F \& Tang G J 2003 Mech. Research Commun. 30, 345-351.

Li Y L, Cross L E \& Chen L Q 2005 J. Appl. Phys. 98, 064101(1-4).

Liu T Q, Oates W S, Wan S \& Lynch C S 2005 J. Intell. Mater. Syst. Struct. 16, 373-379.

Lucato S L D E, Bahr H A, Pham V B, Lupascu D C, Balke H, Rodel J \& Bahr U 2002 J. Mech. Phys. Solids 50, 2333-2353.

Lucato S L D E, Bahr H A, Pham V B, Lupascu D C, Balke H, Rodel J \& Bahr U 2003 J. Eur. Ceram. Soc. 23, 1147-1156.

Lucato S L D E, Lupascu D C, Kamlah M, Rodel J \& Lynch C S 2001 Acta Mater. 49, 2751-2759.

Lynch C S, Chen L, Suo Z, McMeeking R M \& Yang W 1995 J. Intell. Mater. Syst. Struct. 6, 191-198.

Pritchard J, Bowen C R \& Lowrie F 2001 Brit. Ceram. Trans. 100, 265-273.

Ru C Q 2000 J. Mech. Phys. Solids 48, 693-708.

Ru C Q, Mao X \& Epstein M 1998 J. Mech. Phys. Solids 46, 1301-1318.

Shieh J, Huber J E \& Fleck N A 2006 J. Eur. Ceram. Soc. 26, 95-109.

Stemmer S, Streiffer S K, Ernst F \& Ruhle M 1995 Phil. Mag. A 71, 713-724.

Su Y \& Landis C M 2007 J. Mech. Phys. Solids 55, 280-305.

Suo Z 1993 J. Mech. Phys. Solids 41, 1155-1176.

Uchino K \& Furuta A 1992 in M. Liu and A. Safari and A. Kingon And G. Haertling, ed., 'ISAF 92 : Proc. 8th IEEE Int. Symp. Applications of Ferroelectrics' pp. 195-198.

Uchino K \& Takahashi S 1996 Curr. Opin. Solid. State Mater. Sci. 1, 698-705.

Wang J \& Kamlah M 2010 Eng. Fract. Mech. 77, 3658-3669.

Wang Y L, Tagantsev A K, Damjanovic D, Setter N, Yarmarkin V K, Sokolov A I \& Lukyanchuk I A 2007 J. Appl. Phys. 101, 104115(1-9).

Wang Y W \& Mai B L 2005 Sens. Actuat. A: Phys. 121, 203-212.

Westrain I, Oates W S, Lupascu D C, Roedel J \& Lynch C S 2007 Acta Mater. 55, 301-312.

Westram I, Lupascu D, Roedel J, Laskewitz B \& Kamlah M 2007 J. Am. Ceram. Soc. 90, 2849-2854.

Winzer S R, Shankar N \& Ritter A P 1989 J. Am. Ceram. Soc. 72, 2246-2257.

Yang W \& Suo Z 1994 J. Mech. Phys. Solids 42, 649-663.

Ye R Q \& He L H 2001 Int. J. Solids Struct. 38, 6941-6951. 
Young A L, Hilmas G E, Zhang S C \& Schwartz R W 2007 J. Mater. Sci. 42, 5613-5619.

Zhang T Y \& Gao C F 2004 Theor. Appl. Fract. Mech. 41, 339-379.

Zhang T Y, Liu G N \& Wang Y 2004 Acta Mater. 52, 2025-2035.

Zhang W \& Bhattacharya K 2005 Acta Mater. 53, 185-198.

Zhao X J, Liu B \& Fang D N 2010 Int. J. Plast. 26, 533-548. 